\title{
Aneurysms Associated with Brain Arteriovenous Malformations
}

\author{
(D) S.K. Rammos, (D) B. Gardenghi, (DC. Bortolotti, (D).J. Cloft, and DG. Lanzino
}

\begin{abstract}
SUMMARY: Brain arteriovenous malformations are frequently associated with the presence of intracranial aneurysms at a higher-thanexpected incidence based on the frequency of each lesion individually. The identification of intracranial aneurysms in association with AVMs has increased due to improvement in diagnostic techniques, particularly 3D and superselective conventional angiography. Intracranial aneurysms may confer a higher risk of hemorrhage at presentation and of rehemorrhage in patients with AVMs and therefore may be associated with a more unfavorable natural history. The association of AVMs and intracranial aneurysms poses important therapeutic challenges for practicing neurosurgeons, neurologists, and neurointerventional radiologists. In this report, we review the classification and radiology of AVM-associated intracranial aneurysms and discuss their clinical significance and implications for treatment.
\end{abstract}

ABBREVIATION: IA = intracranial aneurysm

B rain arteriovenous malformations are an important cause of intracranial hemorrhage, especially in young individuals, and are associated with increased morbidity and mortality. AVMs are frequently associated with the presence of intracranial aneurysms (IAs) at a higher incidence compared with the anticipated frequency of each lesion individually. Reported rates of IA identification in association with AVMs have increased due to improvement in diagnostic techniques, particularly $3 \mathrm{D}$ and superselective conventional angiography. Intracranial aneurysms may confer a higher risk of hemorrhage at presentation and of rehemorrhage in patients with AVMs and therefore may be associated with a more unfavorable natural history. ${ }^{1-6}$ In this report, we review the classification and radiology of AVM-associated IAs and discuss their clinical significance and implications for treatment.

\section{Classification}

A clear classification scheme and standardized nomenclature of the different IAs encountered in association with AVMs is of paramount importance to guide treatment decision-making. Loca-

From the Department of Neurosurgery (S.K.R.), Arkansas Neuroscience Institute, Little Rock, Arkansas; Institute of Neurosurgery (B.G.), University Hospital of Verona, Verona, Italy; Department of Neurosurgery (C.B.), Istituto Di Ricovero e Cura a Carattere Scientifico, Institute of Neurological Science of Bologna, Bologna, Italy; and Departments of Radiology (H.J.C., G.L.) and Neurosurgery (G.L.), Mayo Clinic, Rochester, Minnesota.

Please address correspondence to Giuseppe Lanzino, MD, Mayo Clinic, 200 First St, Rochester, MN 55905; e-mail: Giuseppe.Lanzino@mayo.edu

- Indicates open access to non-subscribers at www.ajnr.org

http://dx.doi.org/10.3174/ajnr.A4869 tion, underlying hemodynamic features, and histopathology have all been used to classify IAs associated with AVMs. None of the already described classification schemes are widely accepted. Ideally, a classification scheme based on the anatomic and pathophysiologic relationship of the IA to the AVM could have predictive value for the risk of hemorrhage and the potential impact of hemodynamic changes resulting from AVM treatment. Such a system could then evolve, taking into consideration contemporary microsurgical, endovascular, and radiosurgical treatment modalities (Fig 1).

Aneurysms and aneurysm-like dilations can be divided in reference to the AVM nidus into extranidal and intranidal. Extranidal aneurysms are located on the wall of feeding arteries (arterial aneurysms) or on draining veins (venous varices) proximal (prenidal) and distal (postnidal) to the AVM nidus, respectively. Intranidal aneurysms are, by definition, located within the boundaries of the nidus and are angiographically opacified before substantial venous filling has occurred. ${ }^{7}$ Given that pathologic specimens of resected AVM nidi consist of a conglomerate of venous tangles and loops, implicating that venous drainage begins at the level of the nidus, intranidal aneurysms are de facto venous. ${ }^{8}$

Arterial aneurysms may be present on vessels that are not AVM feeders (unrelated aneurysms) or arise from vessels that play a role in the perfusion of the nidus and, as such, are hemodynamically related to the AVM (flow-related aneurysms). Flowrelated arterial aneurysms can occur at a considerable distance from the nidus (proximal flow-related aneurysms) or originate from feeding vessels in close proximity to the nidus (distal flow- 


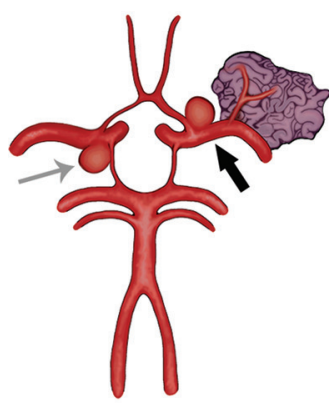

A
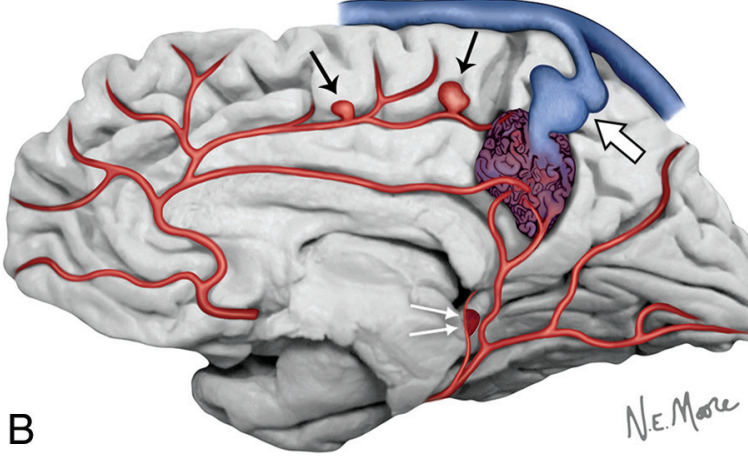

FIG 1. In this circle of Willis figure model (A), an AVM nidus in relation to a branch of the left middle cerebral artery is noted. At the left internal carotid artery bifurcation, a hemodynamically relevant aneurysm is located proximal to the feeding pedicle of the AVM nidus (proximal flowrelated aneurysm) (black arrow). An unrelated aneurysm, with no hemodynamic connection to the AVM nidus, is present at the right posterior communicating artery origin (gray arrow). In this midsagittal view of the brain $(B)$, distal flow-related aneurysms are seen to originate from the feeding arterial pedicles of the AVM nidus (black arrows). Arterial pseudoaneurysms are thought to be the result of the rupture of thin-walled small perforating arteries that supply the AVM and result from the unclotted portion of the hematoma still in communication with the vessel lumen and are very close to the ependymal surface (double white arrows). Finally, venous varices represent irregular, usually circumferential, enlargements of the venous outflow tract of the AVM nidus (large white arrow).

related aneurysms). According to the classification of Redekop et $\mathrm{al}^{7}$ of arterial AVM-associated intracranial aneurysms, flow-related aneurysms should be considered proximal if they are located on the supraclinoid internal carotid artery, the circle of Willis, the middle cerebral artery, up to and including the primary bifurcation, the anterior cerebral artery, up to and including the anterior communicating artery, or the vertebrobasilar trunk. All flow-related aneurysms located distal to the aforementioned bifurcation points are considered distal. While proximal flow-related aneurysms predominantly occur at bifurcations, similar to isolated saccular aneurysms, distal flow-related aneurysms frequently occur along the course of the feeding artery pedicle, not related to bifurcations, and may exhibit irregular shapes and a wide neck.?

The term "venous aneurysm" is, in fact, inaccurate because the wall differs histologically from the arterial aneurysm wall; therefore, the term "venous varices" is preferred. A constellation of venous abnormalities has recently been described due to the use of superselective angiography, including fusiform (circumferential) and narrow-neck (similar morphologically to saccular aneurysms) variceal enlargements. ${ }^{8}$

Garcia-Monaco et al in $1993^{9}$ first described the presence of pseudoaneurysms occurring in AVMs. Arterial pseudoaneurysms most commonly originate from small perforating arteries or choroidal branches in proximity to the ependymal surface. The location of arterial pseudoaneurysms indicates the exact point of rupture and represents a weak point of the wall of the vessel. Pseudoaneurysms may therefore show progressive enlargement on repeat angiography. However, spontaneous regression of such pseudoaneurysms has been observed (Fig 2). Arterial pseudoaneurysms may have an irregular shape, and their formation may be the end result of dissection and dynamic vessel remodeling, as underlined by the presence of persistent filling defects and stenoses. ${ }^{9}$

The real impact of intranidal aneurysms on the hemorrhagic presentation is difficult to verify. The AVM nidus is a complex arrangement of pouches, fistulas, and interconnected circuitry. When an operation is performed shortly after a hemorrhage, venous pseudoaneurysms are encountered as areas of venous dilations, partially filled with thrombus, and indicate the site of rupture at the venous side of shunting.

\section{Pathogenesis and Demographics}

The pathogenesis of IAs in the setting of AVMs is not fully understood. Development of IAs may be related to hemodynamic factors dictated by the presence of shunting in the AVM nidus. This theory is supported by the observation that most aneurysms are located on proximal arteries hemodynamically connected to the AVM nidus. Furthermore, prenidal aneurysms are more frequently encountered in high-flow AVMs and increase in incidence with increasing patient age, a finding that suggests that their nature may be acquired and their formation, the result of the long-term effects of increased flow requirements. Similarly, it is common for prenidal IAs to regress after AVM obliteration. ${ }^{6}$

Only a fraction of patients with AVMs have IAs; therefore, their formation is postulated to be the result of a complex interaction of flow-related factors, host-specific characteristics, and genetic predisposition. ${ }^{7}$ In particular, infratentorial AVMs have been shown to have a higher incidence of associated IAs, hemorrhagic presentation, and unfavorable outcomes. ${ }^{3,4}$ In a recent study, AVMs supplied by the posterior circulation, of which $72 \%$ were perfusing supratentorial malformations, were found to be more commonly associated with IAs. This finding was suggested to be the result of the interaction between the increased hemodynamic stress due to the presence of the malformation itself and greater peak systolic pressure within the vertebrobasilar system, compared with the anterior circulation. ${ }^{10}$ Furthermore, in a comparison of AVM supplying arteries with and without IAs, feeder artery diameter was found to be smaller in feeders with aneurysms, despite similarly high flows on quantitative MR angiography. Arteriovenous malformation feeders with IAs may therefore represent a subgroup in which vessel remodeling cannot compensate for increased blood flow. ${ }^{11}$

On the contrary, in a study that quantified transit times through the AVM nidus as a surrogate of altered hemodynamics, investigators did not find an association of IAs with alterations in AVM hemodynamics. Only a history of prior hemorrhage was shown to correlate with abnormal transit times, leading the authors to conclude that the hemorrhage itself may cause hemodynamic changes and not the other way around. Most interesting, changes in AVM hemodynamics were found to persist long after hemorrhage and did not decrease with time. ${ }^{12}$

The reported incidence of IAs associated with AVMs varies considerably among different studies $(2.7 \%-58 \%) .{ }^{13}$ In a recent meta-analysis on the natural history of brain AVMs, the incidence 


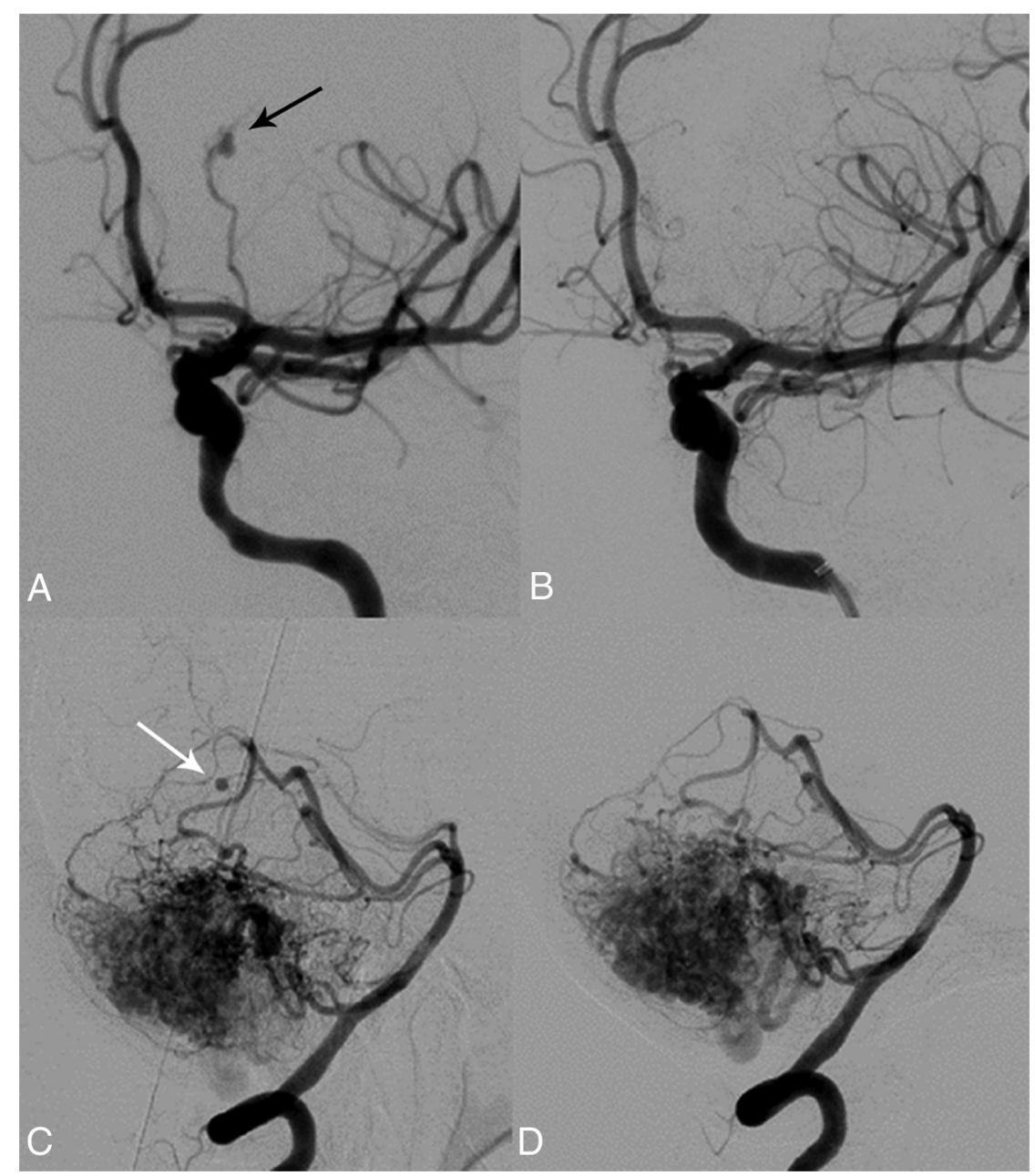

FIG 2. A 46-year-old woman with loss of consciousness and right hemiparesis due to an intracerebral hemorrhage in the left thalamus. Conventional angiography (oblique view) reveals a pseudoaneurysm (black arrow) of a left posterior communicating artery branch that was treated with $N$-butyl cyanoacrylate embolization $(A)$. Complete pseudoaneurysm obliteration after endovascular treatment was achieved $(B)$. Spontaneous regression of a pseudoaneurysm (white arrow) associated with a branch of the right superior cerebellar artery is noted in a 74-year-old woman with subarachnoid hemorrhage centered in the right ambient cistern ( $C$ and $D)$.

of IAs was reported to be $18 \% .{ }^{14}$ A higher incidence of IAs in infratentorial AVMs has also been described. ${ }^{6,15,16}$ Discrepancies in the actual incidence are likely multifactorial, including patient population, use of superselective angiography, and the inclusion of intranidal aneurysms. ${ }^{17}$ Intranidal aneurysms may, in fact, be visualized during embolization procedures as nidus obliteration progresses. In fact, according to one study, the interrater reliability for the diagnosis of coexisting aneurysms in patients with AVMs was only $40 \% .{ }^{18}$ Finally, in the prospective A Randomized Trial of Unruptured Brain AVMs (ARUBA) involving 39 clinical sites in 9 countries, the incidence of AVM-associated (defined as flow-related and located on an AVM feeding artery or intranidal) and unrelated IAs was $16.1 \%$ and $4.9 \%$, respectively, in a series of 223 patients with treated and untreated unruptured AVMs. ${ }^{19}$

\section{Natural History}

In ARUBA, the spontaneous annual hemorrhage rate of previously unruptured AVMs was found to be $2.2 \%{ }^{19}$ Earlier reports similarly estimated the risk of rupture at $3 \%$ per year, while the rehemorrhage rate was $6 \%-15 \%$ for the first year, and thereafter, it approximated the risk of hemorrhage of previously unruptured AVMs. ${ }^{14,20}$

It is commonly cited that the presence of IAs in patients with AVMs is associated with an increased risk of hemorrhage. ${ }^{4,6,7,14,21-23}$ In their meta-analysis, Gross and $\mathrm{Du}^{14}$ calculated that the presence of IAs increased the risk of hemorrhagic presentation by a factor of 1.8. Unrelated aneurysms, because they seem to be randomly associated with AVMs, exhibit a risk of hemorrhage similar to that of saccular aneurysms in the general population, and their presence does not appear to affect the risk of hemorrhage from the AVM. Certain authors have suggested that prenidal aneurysms are more likely to present with hemorrhage compared with intranidal aneurysms, ${ }^{7,20}$ while others have found that distal flow-related and intranidal aneurysms that are immediately adjacent to the site of arteriovenous shunting may be more prone to rupture, given the higher flow, pressure, and shear stress on the vessel wall. ${ }^{8}$ Finally, the presence of venous ectasia has been found to be inversely related to the risk of hemorrhage, probably reflecting a protective adaptive mechanism that may become more prevalent in older patients. ${ }^{24}$

In a landmark study of patients with unruptured AVMs seen at the Mayo Clinic between 1974 and 1985, the risk of hemorrhage among patients with a coexisting, originally unruptured AVM and IA was $7 \%$ at 1 year compared with $3 \%$ among those with an AVM alone. This higher risk of hemorrhage persisted at 5 years in patients with AVMs and IAs (7\%/year) compared with patients with isolated AVMs (1.7\%/year). ${ }^{2}$

On the contrary, the independent association between coexisting IAs in patients with AVMs and presentation with hemorrhage, compared in 2 tertiary referral centers in the United States, was found to differ significantly. Despite sharing remarkably similar AVM features, initial presentation with intracerebral hemorrhage was associated with a coexisting aneurysm in one center, but not in the other. In fact, an opposite trend was noted in the latter center, where an IA associated with the AVM was less likely to be detected in patients initially presenting with intracerebral hemorrhage. This finding underscores the limitations of drawing conclusions from referral-based studies and suggests the potential pitfall of including aneurysm presence in AVM risk-stratification models for patient management and clinical trials. ${ }^{25}$ 


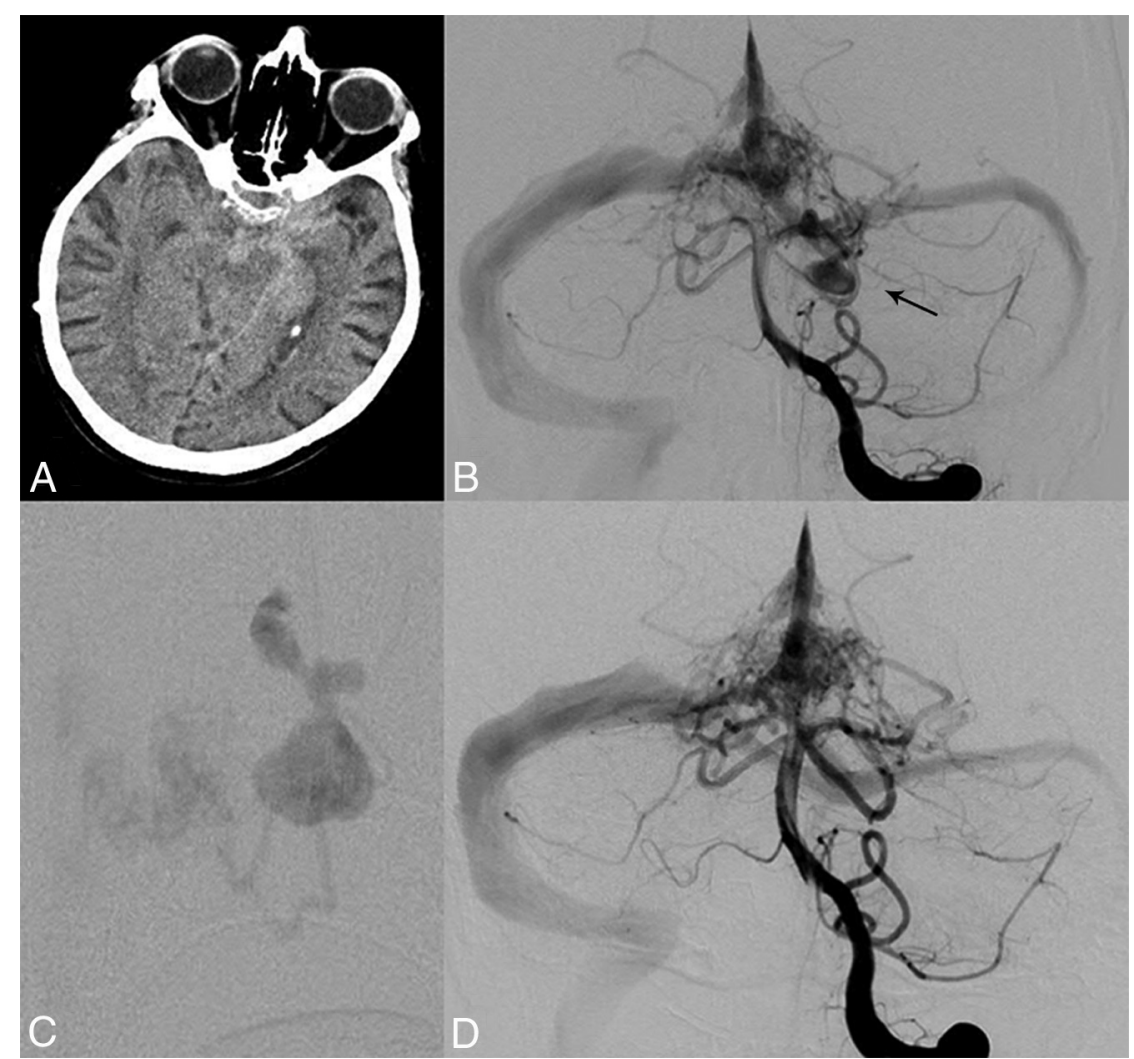

FIG 3. A 70-year-old man with subarachnoid hemorrhage centered in the prepontine cistern (A). Left vertebral artery angiography (anteroposterior view) reveals an AVM of the region of the torcula and a large irregular aneurysm of the left superior cerebellar artery ( $B$ and $C$ ). The presence of isolated subarachnoid hemorrhage suggests the aneurysm as the source of hemorrhage. The aneurysm was treated selectively with $\mathrm{N}$-butyl cyanoacrylate embolization as noted on postprocedural angiography (anteroposterior view) (D), while the treatment of the AVM nidus was deferred.

\section{Treatment}

No consensus currently exists on the treatment of IAs associated with AVMs. While strong evidence may be lacking, advances in microsurgery, endovascular technology and technique, and radiosurgery have expanded the availability of treatment options. Treatment options need to be weighed to optimize the risk-to-benefit profile of the intervention based on the expertise and experience of the institution and treating physicians. Reported AVM treatment complication rates are not negligible for surgery (29\%; range, 1.5\%-54\%), endovascular treatment (25\%; range, $7.6 \%-55 \%)$, and radiosurgery (13\%; range, $0 \%-63 \%)$ as observed in a systematic review of ruptured and unruptured $\mathrm{AVMs}^{26}$ and in the ARUBA study of previously unruptured AVMs (30.7\% complication rate for all treatment modalities). ${ }^{19}$

Any consideration of the treatment of IAs associated with AVMs needs to establish the exact site of rupture in the event of hemorrhagic presentation. The anatomic relationship of IAs and the AVM nidus should be carefully considered when treatment plans are considered. It is of utmost importance to understand whether the source of hemorrhage is the AVM nidus or the IA itself. The diagnosis is made on the basis of clinical experience and inference, and it becomes clearer the farther the hemorrhage is spatially related to the nidus on the head CT scan obtained at presentation. ${ }^{17}$ Correlations can then be made with angiographic studies, including superselective injections. Subarachnoid hemorrhage without associated intracerebral hemorrhage strongly implicates the IA as the possible source of the bleed (Fig 3). Similarly, the presence of a focal hematoma adjacent to the IA with secondary extension to the subarachnoid space implicates the IA as the source of rupture. In the presence of intracerebral hemorrhage with or without $\mathrm{SAH}$, the identification of the hemorrhage epicenter in relation to the nidal angioarchitecture may offer clues to the source of hemorrhage, and dynamic CT performed in the angiography suite may further elucidate the spatial relationship between IAs associated with AVMs and the hemorrhage. ${ }^{27}$

If the IA is considered the source of hemorrhage, then the aneurysm should be treated as early as safely possible following the same treatment criteria for isolated saccular arterial aneurysms. If the aneurysm is located in proximity to the AVM and the AVM itself can be resected surgically, both lesions should be treated simultaneously in a single operation. Proximal flow-related aneurysms should be treated with either surgical or endovascular modalities, depending on their location, morphology, and operator experience. ${ }^{6,13,28}$ Treatment of the associated AVM may be postponed for a later time and can be either surgical, endovascular, radiosurgical, or even conservative (Fig 4). In cases in which the bleeding source is an associated aneurysm and AVM treatment is not indicated, endovascular or surgical closure of the aneurysm alone should be pursued. However, a high rate of aneurysm recurrence has been noted in endovascularly treated IAs without definitive nidal obliteration. $^{6}$

If the source of the hemorrhage is a pseudoaneurysm arising from a small perforating branch, then either prompt treatment or close imaging follow-up should be pursued. Pseudoaneurysms lack a true wall and often display a "dynamic course" in the acute phase with early expansion and rerupture or even spontaneous regression. If the pseudoaneurysm is treated, the AVM can then be treated electively later because the source of hemorrhage has been secured. Endovascular techniques offer a particular advantage when intravascular access can be safely achieved in the vicinity of the pseudoaneurysm, given that most pseudoaneurysms are located on perforating arteries, which can be difficult to reach with an operation.

If the source of hemorrhage has been determined to arise from the AVM or from an intranidal aneurysm, then treatment may not be urgent because the risk of early rerupture is relatively low unless impaired venous outflow of the nidus is present. The lesion can be managed conservatively initially, and an an- 


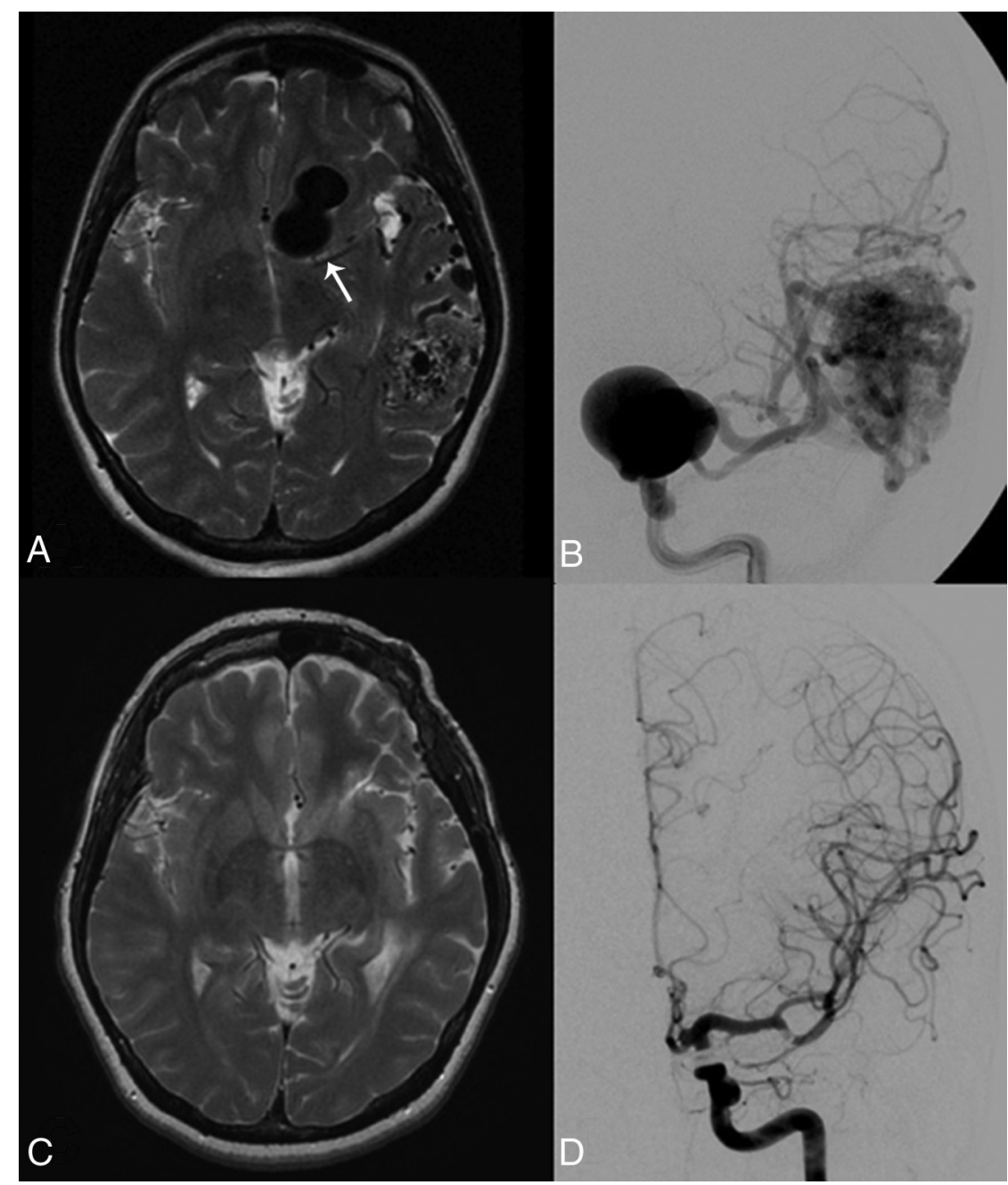

FIG 4. A 42-year-old woman who lost consciousness while dancing. MR imaging (T2 axial) reveals a large flow void suggestive of a giant left aneurysm (arrow) and an associated left temporal lobe AVM (A). Conventional angiography (anteroposterior projection) confirmed a giant left ICA aneurysm and the left temporal AVM (B). The aneurysm was treated with surgical clipping, and the patient underwent stereotactic radiosurgery for the AVM. Follow-up MR imaging (T2 axial) (C) and conventional angiography $(D) 6$ years later show complete exclusion of the aneurysm and obliteration of the AVM.

giogram can be obtained after $4-6$ weeks. Then, if the balance between the risks of any intended procedure and the risk of the natural history of the lesion are favorable, the management of the AVM and the intranidal aneurysm can proceed as an elective case. Preoperative embolization that targets high-flow fistulas and associated prenidal and intranidal IAs before definitive surgery is a valid option, though its effectiveness has not been firmly established. It is unknown at present whether the strategy of palliative embolization of the nidus/feeding pedicle supplying a segment of the AVM harboring an intranidal aneurysm indeed protects the patient from recurrent hemorrhage. $^{29}$

The treatment goal of associated IAs in patients with unruptured AVMs follows concepts similar to those applied to the treatment of unruptured incidental aneurysms in general. However, it has been shown that distal flow-related aneurysms may decrease in size or even disappear after treatment of the AVM. In a study on the course of untreated aneurysms associated with
AVMs after definitive AVM treatment, $80 \%$ of distal flow-related aneurysms regressed after complete AVM occlusion. There were no episodes of SAH from a flow-related aneurysm after AVM obliteration after a follow-up of 7.4 years. On the other hand, of 23 proximal flow-related aneurysms, $78.3 \%$ were unchanged, $17.4 \%$ were smaller, and only $4.3 \%$ were angiographically obliterated after AVM treatment. ${ }^{7}$ Because distal flow-related IAs are likely to regress or decrease in size after complete AVM treatment, conservative management of small distal aneurysms may be considered if the AVM is treated. Furthermore, the rate of hemorrhage after radiosurgery in patients with associated IAs may be significantly increased (28\% at 5 years versus $2.6 \%$ ); therefore, endovascular or microsurgical treatment of IAs should be considered in patients whose AVM nidus is targeted with radiosurgery. ${ }^{30}$

\section{CONCLUSIONS}

Aneurysms associated with intracranial AVMs may confer an increased risk of hemorrhagic presentation. Treatment decisions are based primarily on clinical presentation and the relationship of the IA to the AVM nidus. ${ }^{27}$ In a hemorrhagic clinical presentation, it is critical to establish the source of hemorrhage. If a prenidal aneurysm is considered the source of the hemorrhage, then it should be treated in an expedited fashion, following the same treatment criteria for isolated ruptured saccular aneurysms, with either surgical or endovascular modalities. If the source of the hemorrhage is suspected to arise within the AVM nidus, however, treatment can be delayed because the risk of early rehemorrhage from a ruptured AVM is relatively low as long as there is no severe venous outflow obstruction restricting nidal drainage. In cases in which the source of the hemorrhage is a pseudoaneurysm arising from a small perforating branch, either prompt treatment or close imaging follow-up should be pursued. Treatment of associated IAs in most patients with unruptured AVMs should follow the same principles applied to isolated unruptured aneurysms in the general population. Distal flow-related aneurysms have been shown to regress after definitive AVM treatment; thus, conservative management of small distal flow-related aneurysms may be considered after definitive AVM treatment.

Disclosures: Giuseppe Lanzino_UNRELATED: Consultancy: Covidien/Medtronic.* *Money paid to the institution. 


\section{REFERENCES}

1. Stapf C, Mohr JP, Pile-Spellman J, et al. Concurrent arterial aneurysms in brain arteriovenous malformations with haemorrhagic presentation. J Neurol Neurosurg Psychiatry 2002;73:294-98 CrossRef Medline

2. Brown RD Jr, Wiebers DO, Forbes GS. Unruptured intracranial aneurysms and arteriovenous malformations: frequency of intracranial hemorrhage and relationship of lesions. J Neurosurg 1990;73: 859-63 CrossRef Medline

3. Abla AA, Nelson J, Rutledge WC, et al. The natural history of AVM hemorrhage in the posterior fossa: comparison of hematoma volumes and neurological outcomes in patients with ruptured infraand supratentorial AVMs. Neurosurg Focus 2014;37:E6 CrossRef Medline

4. da Costa L, Thines L, Dehdashti AR, et al. Management and clinical outcome of posterior fossa arteriovenous malformations: report on a single-centre 15-year experience. J Neurol Neurosurg Psychiatry 2009;80:376-79 Medline

5. Gross BA, Ropper AE, Du R. Vascular complications of stereotactic radiosurgery for arteriovenous malformations. Clin Neurol Neurosurg 2013;115:713-17 CrossRef Medline

6. Platz J, Berkefeld J, Singer OC, et al. Frequency, risk of hemorrhage and treatment considerations for cerebral arteriovenous malformations with associated aneurysms. Acta Neurochir (Wien) 2014; 156:2025-34 CrossRef Medline

7. Redekop G, TerBrugge K, Montanera W, et al. Arterial aneurysms associated with cerebral arteriovenous malformations: classification, incidence, and risk of hemorrhage. J Neurosurg 1998;89: 539-46 CrossRef Medline

8. D'Aliberti G, Talamonti G, Cenzato M, et al. Arterial and venous aneurysms associated with arteriovenous malformations. World Neurosurg 2015;83:188-96 CrossRef Medline

9. Garcia-Monaco R, Rodesch G, Alvarez H, et al. Pseudoaneurysms within ruptured intracranial arteriovenous malformations: diagnosis and early endovascular management. AJNR Am J Neuroradiol 1993;14:315-21 Medline

10. Morgan MK, Alsahli K, Wiedmann M, et al. Factors associated with proximal intracranial aneurysms to brain arteriovenous malformations: a prospective cohort study. Neurosurgery 2016;78: 787-92 CrossRef Medline

11. Shakur SF, Amin-Hanjani S, Mostafa H, et al. Hemodynamic characteristics of cerebral arteriovenous malformation feeder vessels with and without aneurysms. Stroke 2015;46:1997-99 CrossRef Medline

12. Illies T, Forkert ND, Saering D, et al. Persistent hemodynamic changes in ruptured brain arteriovenous malformations. Stroke 2012;43:2910-15 CrossRef Medline

13. Flores BC, Klinger DR, Rickert KL, et al. Management of intracranial aneurysms associated with arteriovenous malformations. Neurosurg Focus 2014;37:E11 CrossRef Medline

14. Gross BA, Du R. Natural history of cerebral arteriovenous malformations: a meta-analysis. J Neurosurg 2013;118:437-43 CrossRef Medline

15. Westphal M, Grzyska U. Clinical significance of pedicle aneurysms on feeding vessels, especially those located in infratentorial arterio- venous malformations. J Neurosurg 2000;92:995-1001 CrossRef Medline

16. Lv X, Li Y, Yang X, et al. Characteristics of arteriovenous malformations associated with cerebral aneurysms. World Neurosurg 2011;76: 288-91 CrossRef Medline

17. Kim EJ, Halim AX, Dowd CF, et al. The relationship of coexisting extranidal aneurysms to intracranial hemorrhage in patients harboring brain arteriovenous malformations. Neurosurgery 2004;54: 1349-57; discussion 1357-58 CrossRef Medline

18. Al-Shahi R, Pal N, Lewis SC, et al; AVM Observer Agreement Study Group. Observer agreement in the angiographic assessment of arteriovenous malformations of the brain. Stroke 2002;33:1501-08 CrossRef Medline

19. Mohr JP, Parides MK, Stapf C, et al; international ARUBA investigators. Medical management with or without interventional therapy for unruptured brain arteriovenous malformations (ARUBA): a multicentre, non-blinded, randomised trial. Lancet 2014;383: 614-21 CrossRef Medline

20. Elhammady MS, Aziz-Sultan MA, Heros RC. The management of cerebral arteriovenous malformations associated with aneurysms. World Neurosurg 2013;80:e123-29 CrossRef Medline

21. Marks MP, Lane B, Steinberg GK, et al. Hemorrhage in intracerebral arteriovenous malformations: angiographic determinants. Radiology 1990;176:807-13 CrossRef Medline

22. Thompson RC, Steinberg GK, Levy RP, et al. The management of patients with arteriovenous malformations and associated intracranial aneurysms. Neurosurgery 1998;43:202-11; discussion 211-12 CrossRef Medline

23. Turjman F, Massoud TF, Vinuela F, et al. Aneurysms related to cerebral arteriovenous malformations: superselective angiographic assessment in 58 patients. AJNR Am J Neuroradiol 1994;15:1601-05 Medline

24. Hetts SW, Cooke DL, Nelson J, et al. Influence of patient age on angioarchitecture of brain arteriovenous malformations. AJNR Am J Neuroradiol 2014;35:1376-80 CrossRef Medline

25. Halim AX, Singh V, Johnston SC, et al; UCSF BAVM Study Project. Brain Arteriovenous Malformation. Characteristics of brain arteriovenous malformations with coexisting aneurysms: a comparison of two referral centers. Stroke 2002;33:675-79 CrossRef Medline

26. van Beijnum J, van der Worp HB, Buis DR, et al. Treatment of brain arteriovenous malformations: a systematic review and meta-analysis. JAMA 2011;306:2011-19 CrossRef Medline

27. Gardenghi B, Bortolotti C, Lanzino G. Aneurysms associated with arteriovenous malformations. Contemp Neurosurg 2014;36:1-6 CrossRef

28. Piotin M, Ross IB, Weill A, et al. Intracranial arterial aneurysms associated with arteriovenous malformations: endovascular treatment. Radiology 2001;220:506-13 CrossRef Medline

29. Crowley RW, Ducruet AF, McDougall CG, et al. Endovascular advances for brain arteriovenous malformations. Neurosurgery 2014; 74(suppl 1):S74-82 CrossRef Medline

30. Kano H, Kondziolka D, Flickinger JC, et al. Aneurysms increase the risk of rebleeding after stereotactic radiosurgery for hemorrhagic arteriovenous malformations. Stroke 2012;43:2586-91 CrossRef Medline 